\title{
Magnetically tunable viscoelastic response of soft magnetic nanocomposites with wormlike surfactant micellar matrix
}

\author{
V.S. Molchanov ${ }^{1, *}$, I. A. Klepikov ${ }^{2}$, I. V. Razumovskaya ${ }^{2}$, O. E. Philippova ${ }^{1}$ \\ ${ }^{1}$ Physics Department, Moscow State University, 119991 Moscow, Russia \\ ${ }^{2}$ Moscow State University of Education, 119991 Moscow, Russia \\ *molchan@polly.phys.msu.ru
}

DOI 10.17586/2220-8054-2018-9-3-335-341

\begin{abstract}
Magnetorheological effects in viscoelastic soft magnetic nanocomposites (SMNs) composed of submicron magnetite particles embedded in a network of wormlike micelles (WLMs) of surfactant were studied in a homogeneous magnetic field. In field, the SMNs showed rapid rise of storage and loss moduli by a few orders of magnitude as a result of the ordering of magnetized particles into chain-like or columnar structures. Moreover, solid-like behavior and yield stress of the SMNs were observed. Study of rheological response on periodic switching of field revealed that the initial viscoelasticity of SMNs did not recover completely after removing field, which was attributed to extremely long relaxation time of the WLM network. It was found that the variation of storage and moduli loss was associated with the stepwise change in magnetic field; this can be fitted by two-exponential functions, a characteristic time for the slower process being almost the same as the relaxation time of SMN without field, indicating that this process is essentially determined by the viscoelastic properties of the matrix.
\end{abstract}

Keywords: magnetorheological fluid, viscoelasticity, wormlike micelles of surfactant, magnetic particles.

Received: 28 November 2017

Revised: 11 March 2018

\section{Introduction}

Magnetorheological fluids (MRFs) [1-4] and magnetic elastomers [5,6] or gels [7,8] have attracted increasing attention, because their rheological properties can be changed continuously, rapidly and reversibly, by applying a magnetic field. In the absence of an external magnetic field, the magnetizable particles are randomly dispersed in the medium. When a certain field strength is imposed, the dispersed particles build up the chain-like structures or columns in the field's direction due to an induced magnetic dipole-dipole interaction $[2,3,9,10]$. Owing to the arrangement of particles, the properties (viscoelasticity, yield stress, heat conduction) of the bulk material can be tuned in milliseconds by orders of magnitude. Such smart materials have found many engineering applications providing semi-active control in various devices such as dampers, shock absorbers, torque transducers, clutches, brakes and ultrafine polishing technology [2,5]. They are employed, in particular, in the automobile industry.

High magnetorheological effects can be achieved when particles are close to each other and have large magnetization values [3]. Therefore, it is necessary to provide high volume fraction and particle sizes. For this reason, the submicron particles are considered more promising for soft magnetic nanocomposite preparation. Carbonyl iron and iron oxide particles are widely used in MRFs due to their high magnetic response and availability [11,12], but their density is very high - nearly $8 \mathrm{~g} / \mathrm{cm}^{3}$ and $5 \mathrm{~g} / \mathrm{cm}^{3}$, respectively. This makes it difficult to disperse the submicron- or micron-sized particles in a liquid carrier. To resolve this problem, highly viscous carriers [11] or polymer network matrices $[13,14]$ can be used. However, these carriers deteriorate the mechanical properties of the system in magnetic field because of the restriction of the motion of the particles within the network [14] and the delay rate of the response [13]. To compensate these negative effects, the concentration of particles should be increased considerably (up to 30 vol \%) [14].

Recently, a new type of carrier for magnetic particles was proposed [15]. This carrier is based on soft matrix of WLMs of surfactants. The advantage of such SMNs is the linear viscoelasticity of the WLM network [16-19]. Linear viscoelastic properties of SMNs allow investigation of the dynamic moduli of the system. Also, the WLMs are "living" objects, they can reversibly break under stress $[18,20,21]$ that makes them promising as a matrix where particles can move freely enough even at low particle concentrations. This shows that WLMs are perfect for the stabilization of particle suspensions, because they are able to link to the particles by their end-caps. However, the rheological behavior of the SMNs was studied mainly in the absence of magnetic field.

Therefore, the present paper is devoted to the investigation of the rheological properties of SMNs composed of submicron magnetite particles embedded in the network of WLMs in magnetic field. The WLMs were built by cationic surfactant erucyl bis-(hydroxyethyl)methylammonium chloride (EHAC) in the presence of low-molecularweight salt $\mathrm{KCl}$. EHAC containing a long mono-unsaturated $\mathrm{C} 22$ tail is known to impart high viscosity to aqueous 
solutions even at rather low surfactant concentrations. To provide stronger interaction with particles [22], the experiments were performed at $\mathrm{pH} 11$, when magnetite particles were negatively charged [15].

\section{Experimental section}

\subsection{Materials}

The cationic surfactant EHAC $\mathrm{C}_{8} \mathrm{H}_{17} \mathrm{CH}=\mathrm{CHC}_{12} \mathrm{H}_{24} \mathrm{~N}\left(\mathrm{CH}_{2} \mathrm{CH}_{2} \mathrm{OH}\right)_{2} \mathrm{CH}_{3} \mathrm{Cl}$ was provided by Akzo Nobel as a solution in 2-propanol. EHAC powder was obtained by freeze drying this solution. ${ }^{1} \mathrm{H}$ NMR spectroscopy data proved the absence of 2-propanol in EHAC thus purified. Potassium chloride (purity $>99 \%$ ) from Acros Organics was used without further purification. Distilled water was obtained with a Milli-Q system (Millipore).

Magnetite particles iron (II,III) oxide $\mathrm{Fe}_{3} \mathrm{O}_{4}$ were supplied by Sigma-Aldrich. By scanning electron microscopy, it was shown that the average diameter of the particles is about $250 \mathrm{~nm}$ [15]. These particles possess ferrimagnetic properties [23], their saturation magnetization is $100 \mathrm{emu} / \mathrm{g}$, the residual magnetization is $19 \mathrm{emu} / \mathrm{g}$, and the coercive force - 95 Oe [15]. Dispersions of these magnetic particles in WLM viscoelastic solutions were prepared following a previously developed procedure [15]. The homogeneity of the suspensions was examined by visual observation after 2 months storage at $25{ }^{\circ} \mathrm{C}$.

\subsection{Rheological Measurements}

The dynamic and static controlled stress rheological measurements of the SMNs in the absence and in the presence of a homogeneous magnetic field were carried out with a commercial rheometer Physica MCR 301 (Anton Paar, Austria). The temperature was fixed at $20{ }^{\circ} \mathrm{C}$. The samples were placed between rotated cone or plate and static plate. Static rheological measurements were performed at shear stress from 0.009 Pa to $100 \mathrm{~Pa}$. Zero-shear viscosity was obtained from plateau of flow curve while yield stress was obtained from stress-strain curve. Dynamic frequency-dependent measurements were performed at frequencies from 0.01 to $10 \mathrm{rad} / \mathrm{s}$ and a fixed strain amplitude within the linear viscoelastic region. Latter was measured at frequency of $1 \mathrm{rad} / \mathrm{s}$ and a strain amplitude from 0.05 to $10 \%$. The measuring system's geometries were a cone-plate set with diameter $50 \mathrm{~mm}$ and angle $1^{\circ}$ for tests without the field because lower shear stress and a parallel-plate set with $20 \mathrm{~mm}$ diameter as part of a specific magnetic equipment. The gap thickness in the latter cell was $300 \mu \mathrm{m}$ in all measurements [24]. The magnetic field with the strength of up to $1 \mathrm{~T}$ was applied in the direction perpendicular to the shear stress $27-23$.

\section{Results and discussion}

Fig. 1 allows one to compare the values of the storage $G^{\prime}$ and loss $G^{\prime \prime}$ moduli for some SMNs before and after the application of magnetic field. It is seen that in the absence of magnetic field, the SMN sample containing 0.2 vol \% magnetite has a storage modulus $\mathrm{G}^{\prime}$ of $0.2 \mathrm{~Pa}$ and a loss modulus $\mathrm{G}^{\prime \prime}$ of $0.02 \mathrm{~Pa}$. When $1 \mathrm{~T}$ magnetic field is applied, $G^{\prime}$ and $G^{\prime \prime}$ moduli increase by 2 orders of magnitude (Fig. 1). This can be explained by the formation of chain-like structures of particles along the magnetic field lines oriented perpendicular to the direction of flow [2,3,25]. Among the chain-like structures, one can distinguish long ones, which spread from one plate of the measuring cell to the other, and shorter ones possessing at least one free end. The long structures attached to both measuring plates are suggested to contribute to the storage modulus $\mathrm{G}^{\prime}$ thus enhancing the elasticity of the system, whereas short structures give input into the loss modulus $\mathrm{G}^{\prime \prime}$ thus increasing the viscous contribution.

In the absence of magnetic field, the SMN with 3 vol \% magnetite has almost the same value of storage modulus $G^{\prime}$ as the sample with 0.2 vol \% magnetite (data not shown here), which is expected, because at these concentrations, the dependencies of $G^{\prime}$ and $G^{\prime \prime}$ on the content of the particles level off [15]. However, in $1 \mathrm{~T}$ magnetic field the values of $G^{\prime}$ and $G^{\prime \prime}$ for the SMN sample with 3 vol \% of particles are by two orders of magnitude higher than for the sample with $0.2 \mathrm{vol} \%$ of particles. It indicates to increasing input of both long and short chain-like structures into viscoelastic properties of SMNs, when the amount of particles making up these structures increases.

Let us consider the evolution of storage $G^{\prime}$ and loss $G^{\prime \prime}$ moduli with increasing mechanical strain depicted in Fig. 1. One can see that in the absence of magnetic field, the SMN sample containing 0.2 vol \% magnetite demonstrates a linear viscoelasticity at all studied strain values up to $10 \%$. This feature of the SMNs is provided by viscoelastic matrix composed of entangled WLMs [19]. When $1 \mathrm{~T}$ magnetic field is applied, the sample continues to exhibit a wide linear viscoelastic region, similar to that observed without the field. However, SMN with higher volume fraction of particles $(0.3 \mathrm{vol} \%)$ has a shorter range of linear viscoelasticity (up to the strain of nearly $1 \%$ ). At higher strains, storage modulus $G^{\prime}$ starts to decrease, indicating the appearance of nonlinearity. It can be attributed to the reorganization (for instance, partial destruction) of the chain-like structures of particles formed in the magnetic field, when the mechanical deformations become rather high $[26,27]$. However, the deviations from 


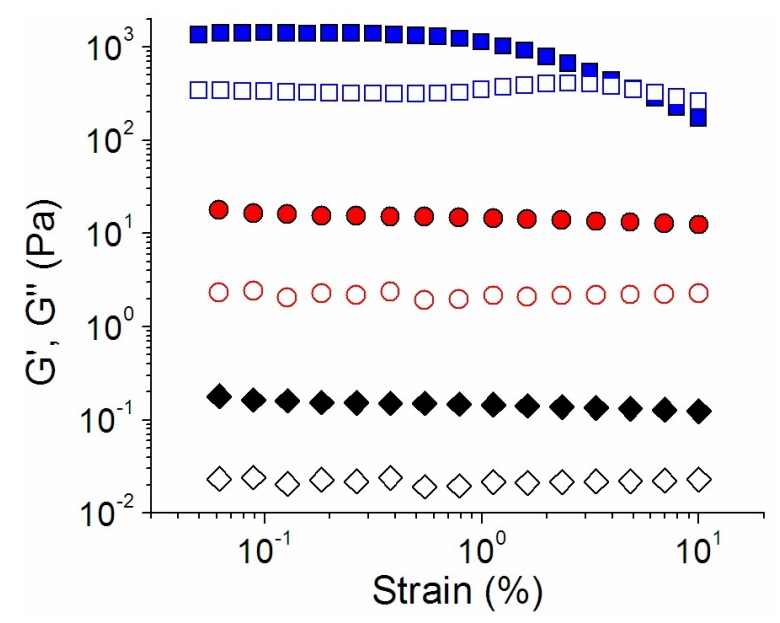

FIG. 1. Dependence of storage $G^{\prime}$ (filled symbols) and loss $G^{\prime \prime}$ (open symbols) moduli on mechanical strain (at $1 \mathrm{rad} / \mathrm{s}$ frequency) for SMN containing $0.2 \mathrm{vol} \%$ magnetite and $0.6 \mathrm{wt} \%$ EHAC without field (black diamonds) and at magnetic field strength of $1 \mathrm{~T}$ (red circles) and for SMN containing $3 \mathrm{vol} \%$ magnetite and $0.6 \mathrm{wt} \%$ EHAC at magnetic field strength of $1 \mathrm{~T}$ (blue squares). Solvent: $1.5 \mathrm{wt} \% \mathrm{KCl}$ in water

linear viscoelasticity occur at rather large strains, which are much higher than in MRFs based on Newtonian liquids $(0.003-0.1 \%[8,12,23])$. Therefore, the SMNs under study show a rather wide range of linear viscoelasticity even at magnetic field strength as high as $1 \mathrm{~T}$. This feature is quite important since the dynamic rheological data obtained within linear viscoelastic region characterize the structure undisturbed by mechanical strain [11], and these data are most suitable for further investigation of the structure transformations induced by magnetic field. Therefore, in the present system, a broad range of linear viscoelastic response within a wide range of deformations (Fig. 1) permits to perform the oscillatory measurements under optimum conditions.

Figure 2 shows the frequency dependences of storage $G^{\prime}(\omega)$ and loss $G^{\prime \prime}(\omega)$ moduli obtained at fixed strain in linear viscoelasticity region. It is seen that without field, the loss modulus has a minimum and the $G^{\prime \prime}(\omega)$ curve as a tendency to intercept with $G^{\prime}(\omega)$ curve so that at lower frequencies loss modulus is expected to exceed the storage modulus. Upon application of magnetic field, the $G^{\prime}$ and $G^{\prime \prime}$ values increase by several orders of magnitude and become independent of frequency (Fig. 2).

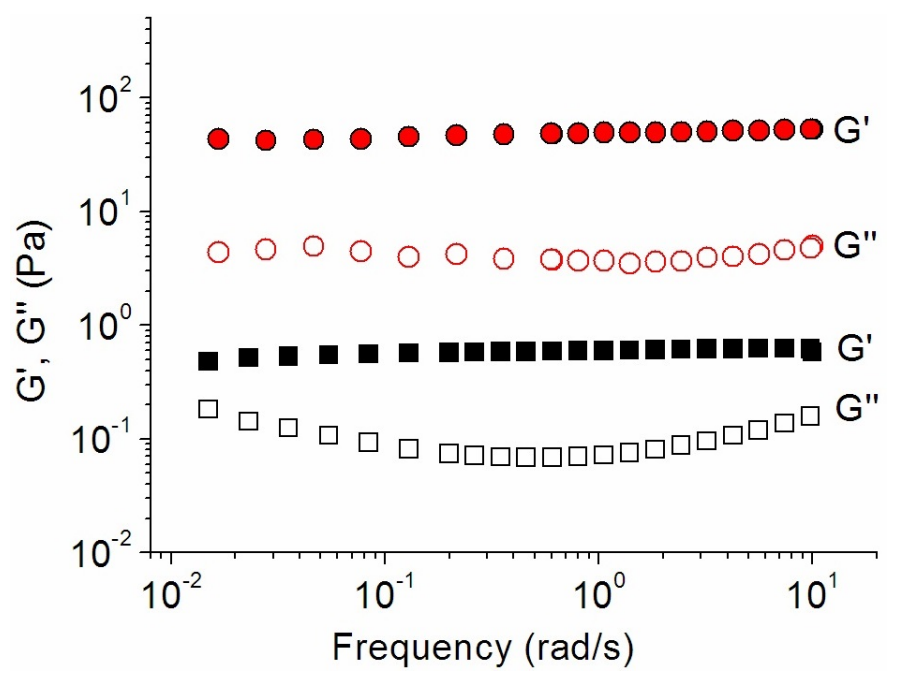

FIG. 2. Frequency dependences of storage (filled symbols) and loss (open symbols) moduli without field (black squares) and in $0.2 \mathrm{~T}$ magnetic field (red circles) at constant strain of $1 \%$ for SMN containing 0.6 vol \% magnetite and $0.6 \mathrm{wt} \%$ EHAC. Solvent: $1.5 \mathrm{wt} \% \mathrm{KCl}$ in water 

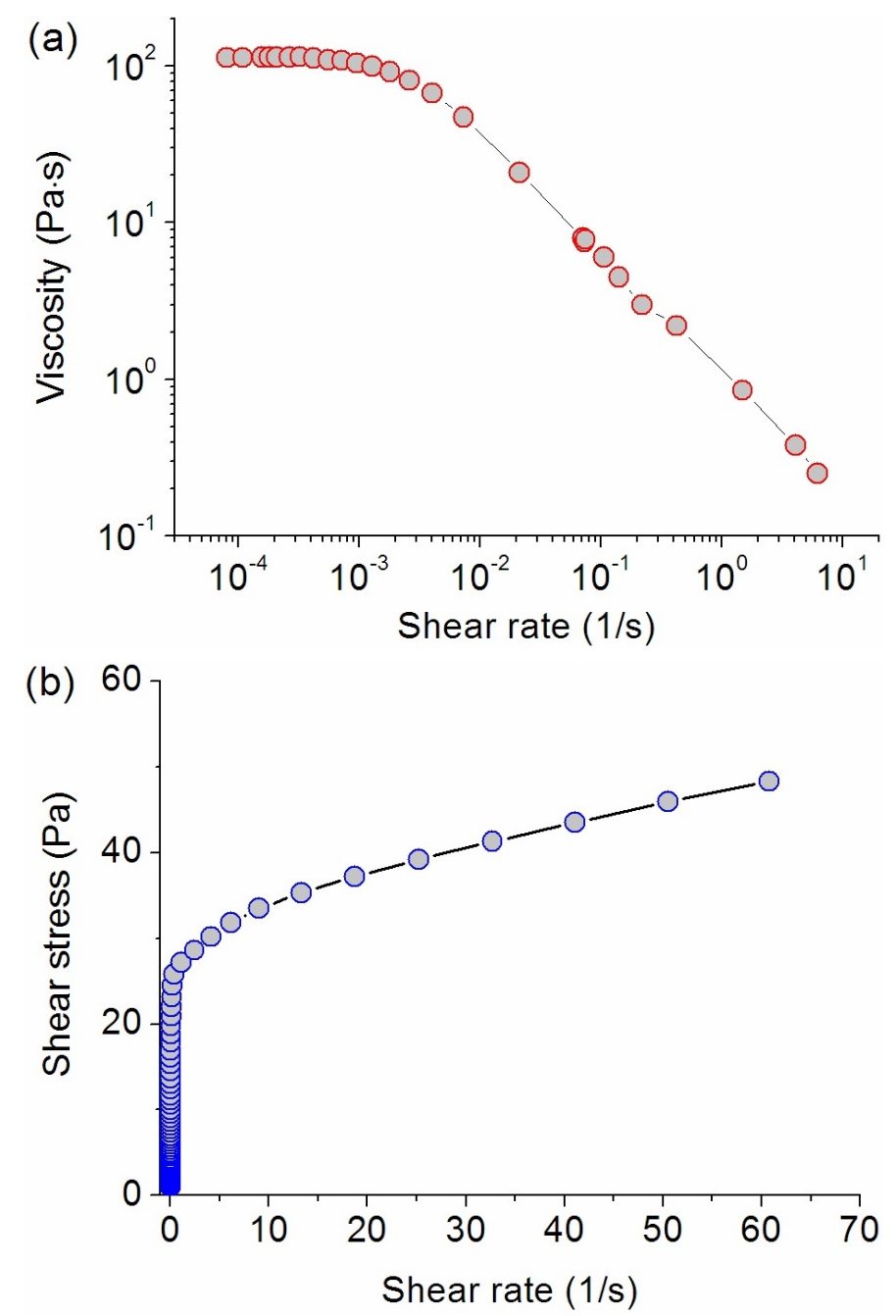

FIG. 3. Viscosity (a) and shear stress (b) plotted as a function of shear rate for SMN sample containing 0.6 vol \% magnetite and $0.6 \mathrm{wt} \%$ EHAC without field (a) and in $0.2 \mathrm{~T}$ magnetic field (b). Solvent: 1.5 wt \% $\mathrm{KCl}$ in water

The results of steady-state rheological measurements are presented in Fig. 3. It is seen that in the absence of magnetic field (Fig. 3a) the SMN shows a flow curve with a shear thinning behavior and a plateau at low shear rates giving the value of zero-shear viscosity. The shear-thinning behavior can be attributed to the alignment of WLMs along the direction of flow [16]. By contrast, in the presence of magnetic field $(0.2 \mathrm{~T})$, the sample does not flow below some critical stress (Fig. 3b). It indicates that upon application of the magnetic field the system acquires a yield stress [28-30]. For the SMN sample with 3 vol \% magnetite and 0.6 wt \% EHAC the yield stress is equal to $24 \mathrm{~Pa}$. Thus, under the action of magnetic field the SMN undergoes a transformation from viscoelastic to a solid-like state.

Figure 4 shows the response of storage $G^{\prime}$ and loss $G^{\prime \prime}$ moduli in oscillatory testing of SMN sample under periodical switching of the $0.2 \mathrm{~T}$ magnetic field every $300 \mathrm{~s}$. In this experiment, the $G^{\prime}$ and $G^{\prime \prime}$ values were determined at a fixed frequency of shear strain equal to $1 \mathrm{rad} / \mathrm{s}$. From Fig. 4 it is seen that the moduli increased synchronically with the magnetic field, but did not fully recover the original values after switching the field off. During several cycles of periodical application of magnetic field the storage $G^{\prime}$ and loss $G^{\prime \prime}$ moduli under the field are the same, whereas without field the residual values of $G^{\prime}$ and $G^{\prime \prime}$ increase with time and finally level off (Fig. 4). The effect can be explained by the impact of the viscoelastic network of WLMs. Indeed, the micelles attached to particles have to be stretched when particles move in order to self-organize in the chain-like or column structures because latter process is quite rapid. Then, WLMs move in direction of the structures of particles and reorganize around new chain-like or column structures. After removing field particles have tendency to disperse randomly [13] but the reorganized WLM network formed around chains/columns retain them in partly ordered 


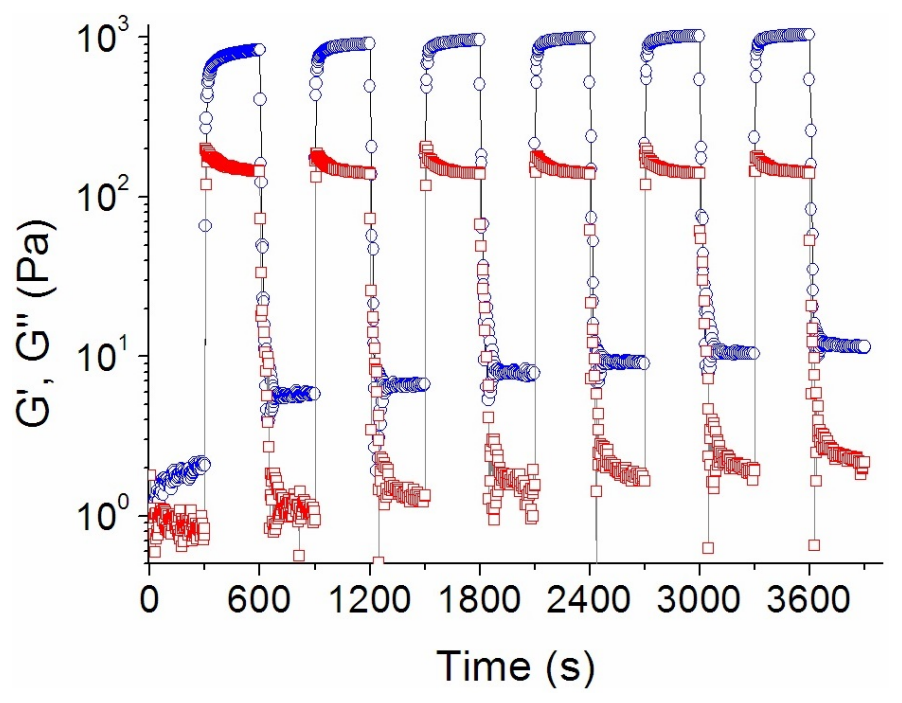

FIG. 4. Evolution of storage $G^{\prime}$ (blue circles) and loss $G^{\prime \prime}$ (red squares) moduli with time in response to periodical switching of $0.2 \mathrm{~T}$ magnetic field for SMN sample containing 0.3 vol \% magnetite and $0.6 \mathrm{wt} \%$ EHAC. Solvent: $1.5 \mathrm{wt} \% \mathrm{KCl}$ in water. $G^{\prime}$ and $G^{\prime \prime}$ values were determined at $1 \mathrm{rad} / \mathrm{s}$ frequency of shear strain

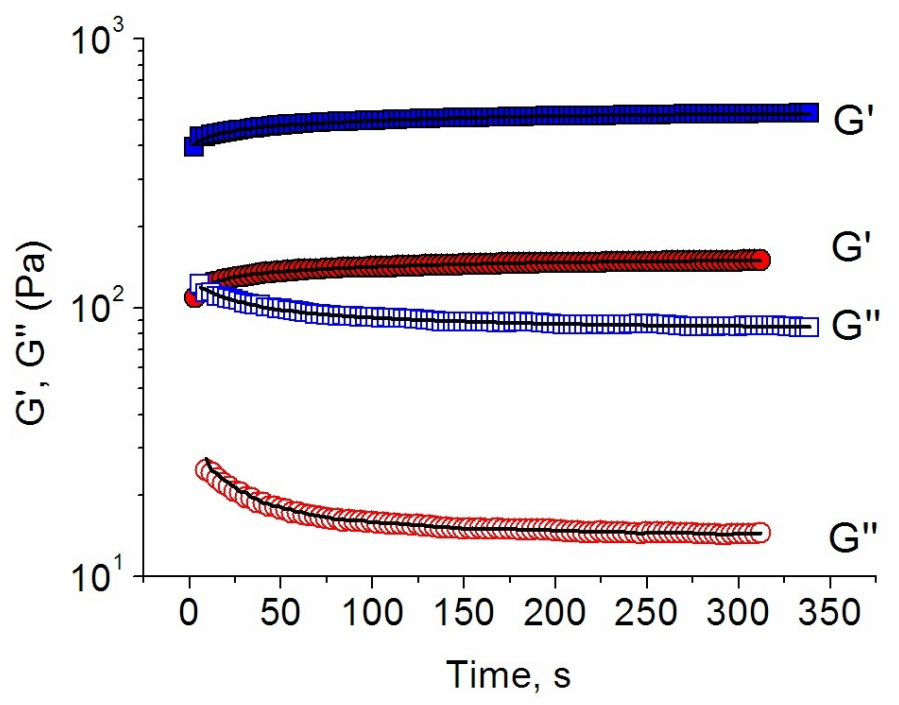

FIG. 5. Evolution of the storage $\mathrm{G}^{\prime}$ (filled) and loss $\mathrm{G}^{\prime \prime}$ (open) moduli with time in magnetic field of $0.04 \mathrm{~T}$ (blue squares) and of $0.07 \mathrm{~T}$ (red circles) for SMN sample containing 0.3 vol \% magnetite and $0.6 \mathrm{wt} \%$ EHAC. Solvent: $1.5 \mathrm{wt} \% \mathrm{KCl}$ in water. $G^{\prime}$ and $G^{\prime \prime}$ values were determined at $1 \mathrm{rad} / \mathrm{s}$ frequency of shear strain. Curves fitting $G^{\prime}(t)$ and $G^{\prime \prime}(t)$ dependences by two-exponential function are depicted by solid lines

conditions. After certain cycles of repeated magnetic field action, the WLMs organize a network around the structures of particles, which is responsible for the residual values of moduli being one order of magnitude higher than the original moduli without field. Thus, with periodical switching of magnetic field the viscoelasticity of the WLM network produces enhanced $G^{\prime}$ and $G^{\prime \prime}$ moduli, which do not acquire their original values. It can be suggested that the system needs longer time to fully recover the initial state because of extremely long relaxation time of the WLM network, especially with embedded particles [15].

Figure 5 displays the change in storage and loss moduli associated with the stepwise change in magnetic field of $0.04 \mathrm{~T}$ and $0.07 \mathrm{~T}$. It is seen that the storage $G^{\prime}$ and loss $G^{\prime \prime}$ moduli response fits well to the two-exponential increase and decrease, correspondingly. The fitting for both moduli results in two values of characteristic time: a shorter one $t_{1}=15 \pm 5 \mathrm{~s}$ and a longer one $t_{2}=110 \pm 10 \mathrm{~s}$. In literature, the fast time was associated with 
the formation of particles having chain-like structures and the slower time was attributed to the migration of these chains into thicker clusters [13]. Also, it was shown that the characteristic time $t_{2}$ for the slow process is essentially determined by the viscoelastic properties of the matrix [13], which is in agreement with our data, since the $t_{2}$ value is almost the same as the relaxation time for SMN without field [15].

\section{Conclusions}

This paper describes the variation of rheological properties of new SMNs upon application of a homogeneous magnetic field. It was shown that the studied SMNs possess very large linear viscoelasticity regions, which allows one to perform dynamic oscillatory shear tests over a wide range of applied mechanical deformations. Such tests are important for modeling the behavior of magnetorheological system under oscillatory shear stress conditions, for instance, in damper applications for vibration control. Despite this fact, such oscillatory tests are rarely used for studying MRFs because the linear viscoelastic region for most of them is limited to extremely low deformations. Periodical switching of magnetic field demonstrated that at each cycle the $G^{\prime}$ and $G^{\prime \prime}$ values in the magnetic field are higher by several orders of magnitude than in its absence. The data obtained show that the new SMNs are very promising for various magnetorheological applications under dynamic conditions.

\section{Acknowledgements}

This work was supported by Russian Foundation for Basic Research and Moscow city Government (project No 15-33-70046 “mol_a_mos").

\section{References}

[1] Odenbach S. Recent Progress in Magnetic Fluid Research. J. Phys.: Condens. Matter, 2004, 16, P. R1135-R1150.

[2] Park B.J., Fang F.F., Choi H.J. Magnetorheology: Materials and Application. Soft Matter, 2010, 6, P. $5246-5253$.

[3] De Vicente J., Klingenberg D.J., Hidalgo-Alvarez R. Magnetorheological Fluids: a Review. Soft Matter, 2011, 7, P. 3701-3710.

[4] Barabanova A., Molchanov V., Philippova O., Khokhlov A., Magnetorheological Fluids Based on Associating Polymers. Macromol. Symp., 2014, 337, P. 80-86.

[5] Filipcsei G., Csetneki I., Szilgyi A., Zrinyi M. Magnetic Field-Responsive Smart Polymer Composites. In Advances in Polymer Science, Oligomers, Polymer Composites, Molecular Imprinting, Springer-Verlag, Berlin, 2007, P. 137-189.

[6] Molchanov V.S., Stepanov G.V., Vasiliev V.G., Kramarenko E.Yu., Khokhlov A.R., Xu Z.-D., Guo Y.-Q. Viscoelastic Properties of Magnetorheologicla Elastomers for Damping Applications. Macromol. Mater. Eng., 2014, 299, P. 1116-1125.

[7] Zhou Y., Sharma N., Deshmukh P., Lakhman R.K., Jain M., Kasi R.M. Hierarchically Structured Free-Standing Hydrogels with Liquid Crystalline Domains and Magnetic Nanoparticles as Dual Physical Cross-Linkers. J. Am. Chem. Soc., 2012, 134, P. 1630-1641.

[8] An H.-N., Sun B., Picken S.J., Mendes E. Long Time Response of Soft Magnetorheological Gels. J. Phys. Chem. B, 2012, 116, P. 47024711.

[9] McLeish T.C.B., Jordan T., Shaw M.T. Viscoelastic Response of Electrorheological Fluids. I. Frequency dependence. J. Rheol., 1991, 35, P. $427-448$.

[10] Ginder J.M., Davis L.C., Elie L.D. Rheology of Magnetorheological Fluids: Models and Measurements. Int. J. Mod. Phys. B, 1996, 10, P. 3293-3303.

[11] Claracq J., Sarrazin J., Montfort J.-P. Viscoelastic Properties of Magnetorheological Fluids. Rheol. Acta, 2004,43 , P. 38-49.

[12] De Vicente J., Segovia-Gutiérrez J.P., Andablo-Reyes E., Vereda F., Hidalgo-Alvarez, R. Dynamic Rheology of Sphere- and Rod-Based Magnetorheological Fluids. J. Chem. Phys., 2009, 131, P. 194902.

[13] An H.-N., Sun B., Picken S.J., Mendes E. Long Time Response of Soft Magnetorheological Gels. J. Phys. Chem. B, 2012, 116, P. 47024711.

[14] Mitsumata T., Honda A., Kanazawa H., Kawai M. Magnetically Tunable Elasticity for Magnetic Hydrogels Consisting of Carrageenan and Carbonyl Iron Particles. J. Phys. Chem. B, 2012, 116, P. 12341-12348.

[15] Pletneva V.A., Molchanov V.S., Philippova O.E. Viscoelasticity of Smart Fluids Based on Wormlike Surfactant Micelles and Oppositely Charged Magnetic Particles. Langmuir, 2015, 31, P. 110-119.

[16] Magid L.J. The Surfactant-Polyelectrolyte Analogy. J. Phys. Chem. B, 1998, 102, P. 4064-4074.

[17] Oda R., Narayanan J., Hassan P.A., Manohar C., Salkar R.A., Kern F., Candau S.J. Effect of the Lipophilicity of the Counterion on the Viscoelasticity of Micellar Solutions of Cationic Surfactants. Langmuir, 1998, 14, P. 4364-4572.

[18] Molchanov V.S., Philippova O.E. Dominant Role of Wormlike Micelles in Temperature-Responsive Viscoelastic Properties of their Mixtures with Polymeric Chains. J. Colloid Interface Sci., 2013, 394, P. 353-359.

[19] Kwiatkowski A.L., Molchanov V.S., Orekhov A.S., Vasiliev A.L., Philippova O.E. Impact of Salt Co- and Counterions on Rheological Properties and Structure of Wormlike Micellar Solutions. J. Phys. Chem. B, 2016, 120, P. 12547-12556.

[20] Shibaev A.V., Tamm M.V., Molchanov V.S., Rogachev A.V., Kuklin A.I., Dormidontova E.E., Philippova O.E. How a Viscoelastic Solution of Wormlike Micelles Transforms into a Microemulsion upon Absorption of Hydrocarbon: New Insight. Langmuir, 2014, 30, P. $3705-3714$.

[21] Philippova O.E., Chtcheglova L.A., Karybiants N.S., Khokhlov A.R. Two Mechanisms of Gel/Surfactant Binding. Polymer Gels Networks, 1998, 6, P. 409-421.

[22] Kwiatkovski A.L., Sharma H., Molchanov V.S., Orekhov A.S., Vasiliev A.L., Dormidontova E.E., Philippova O.E. Wormlike Surfactant Micelles with Embedded Polymer Chains. Macromolecules, 2017, 50, P. 7299-7308. 
[23] Chin B.D., Park J.H., Kwon M.H., Park O.O. Rheological Properties and Dispersion Stability of Magnetorheological (MR) Suspensions. Rheol. Acta, 2001, 40, P. 211-219.

[24] Martin J.M., Odinek J. Evolution of Structure in Quiescent Electrorheological Fluid. Phys. Ref. Lett., 1992, 69, P. 1524-1528.

[25] Otsubo Y. Electrorheological Properties of Barium Titanate Suspensions under Oscillatory Shear. Colloids Surf., 1991, 58, P. 73-86.

[26] Parthasarathy M., Ahn K.H., Belongia B., Klingenberg D.J. The Role of Suspension Structure in the Dynamic Response of Electrorheological Suspensions. Int. J. Mod. Phys. B, 1994, 8, P. 2789-2809.

[27] López-López M.T., Rodriguez-Arco L., Zubarev A., Iskakova L., Durán D.G. Effect of Gap Thickness on the Viscoelasticity of Magnetorheological Fluids. J. Appl. Phys., 2010, 108, P. 083503.

[28] Viota J.L., Delgado A.V., Arias J.L., Durán J.D.G. Study of the Magnetorheological Response of Aqueous Magnetite Suspensions Stabilized by Acrylic Acid Polymers. J. Colloid Interface Sci., 2008, 324, P. 199-204.

[29] Li W.H., Chen G., Yao S.H. Viscoelastic Properties of MR Fluids. Smart Mater. Struct., 1999, 8, P. $460-468$.

[30] López-López M.T., Gómez-Ramirez A., Rodriguez-Arco L., Durán D.G., Iskakova L., Zubarev A. Colloids on the Frontier of Ferrofluids. Rheological Properties. Langmuir, 2012, 28, P. 6232-6245. 